\title{
No association between current depression and latent toxoplasmosis in adults
}

\author{
${ }^{1}$ Department of Psychology, Brigham Young University, Provo, Utah, USA; \\ ${ }^{2}$ The Neuroscience Center, Brigham Young University, Provo, Utah, USA; \\ ${ }^{3}$ Department of Sociology, Brigham Young University, Provo, Utah, USA
}

Shawn D. Gale ${ }^{1,2}$, Andrew N. Berrett ${ }^{1}$, Bruce Brown ${ }^{1}$, Lance D. Erickson ${ }^{3}$ and Dawson W. Hedges ${ }^{1,2}$

\begin{abstract}
Changes in behaviour and cognition have been associated with latent infection from the apicomplexan protozoan Toxoplasma gondii (Nicolle et Manceaux, 1908) in both animal and human studies. Further, neuropsychiatric disorders such as schizophrenia have also been associated with latent toxoplasmosis. Previously, we found no association between T. gondii immunoglobulin G antibody (IgG) seropositivity and depression in human adults between the ages of 20 and 39 years $(n=1846)$ in a sample representative of the United States collected by the Centers for Disease Control as part of a National Health and Nutrition Examination Survey (NHANES) from three datasets collected between 1999-2004. In the present study, we used NHANES data collected between 2009 and 2012 that included subjects aged 20 to 80 years $(n=5487)$ and used the Patient Health Questionnaire 9 (PHQ-9) to assess depression with the overall aim of testing the stability of the results of the prior study. In the current study, the seroprevalence of $T$. gondii was $13 \%$. The percentage of subjects reporting clinical levels of depression assessed with the PHQ- 9 was $8 \%$. As before, we found no association between $T$. gondii $\mathrm{IgG}$ seroprevalence and depression ( $\mathrm{OR}=1.01,95 \% \mathrm{CI}=0.81-1.25 ; p=0.944)$ while controlling for sex, educational attainment, race-ethnicity, age, poverty-to-income ratio and cigarette smoking. We also found no positive associations between anti- $T$. gondii antibody titre and depression $(\mathrm{OR}=1.00,95 \% \mathrm{CI}=0.96-1.06 ; p=0.868)$. Moreover, we found no association between $T$. gondii seroprevalence or antibody titre and suicidal ideation (seroprevalence: $\mathrm{OR}=1.22,95 \% \mathrm{CI}=.85-1.75 ; p=0.277$, titre: $\mathrm{OR}=1.05,95 \% \mathrm{CI}=0.98-1.14 ; p=0.177$ ). Defining depression to also include subjects currently taking antidepressant medication even with non-elevated questionnaires did not find evidence of a positive association between latent toxoplasmosis and depression. In the present study, neither $T$. gondii seroprevalence nor anti- $T$. gondii antibody titre was positively associated with depression or suicidal ideation among subjects aged 20 to 80 years.
\end{abstract}

Keywords: Toxoplasma gondii, major depressive disorder, NHANES

Latent infection with the apicomplexan parasite Toxoplasma gondii (Nicolle et Manceaux, 1908) has been associated with changes in behaviour, cognition and mood (Flegr 2015). While the exact mechanism by which latent toxoplasmosis affects behaviour is unclear, T. gondii can exist intracellularly in the brain throughout the life of the host (Carruthers and Suzuki 2007), potentially modifying neural structure or function (Berenreiterová et al. 2011, Prandovszky et al. 2011, Fuks et al. 2012). Indeed, T. gondii appears to alter dopamine metabolism (Parlog et al. 2015), with the potential to substantially increase dopamine concentration in the brain (Berenreiterová et al. 2011).

The strongest relation between latent toxoplasmosis and behaviour in humans is the association with schizophrenia (Torrey et al. 2007). In a recent meta-analysis, Sutterland et al. (2015) found that after controlling for publication bias, the odds ratio for the association between toxoplasmosis infection and schizophrenia was $1.43(95 \% \mathrm{CI}=1.21-1.70)$.
Despite the considerable research interest in the association between latent toxoplasmosis and schizophrenia, comparatively little research has investigated associations between latent toxoplasmosis and depression (Fekadu et al. 2010), even though in 2014 an estimated 15.7 million adults in the United States (18 years and older) had at least one major depressive episode in the past year (U.S. Department of Health and Human Services 2014).

Further, the results of the available studies investigating the association between latent toxoplasmosis and depression have been inconsistent. Duffy et al. (2015) found higher depression scores on self-report measures in women veterans positive for $T$. gondii as well as correlations between test scores and anti-T. gondii titres. Another study found an association between anti-T. gondii IgG titre levels and use of antidepressants (Hinze-Selch et al. 2007) and another study found higher anti-T. gondii titres in those with a history of recurrent depression and a suicide attempt 
compared to those with a history of recurrent depression without a suicide attempt (Arling et al. 2009).

In another study, the seroprevalence of anti-T. gondii antibodies in subjects who had attempted suicide was higher (41\%) than in healthy controls (28\%) (Yagmur et al. 2010). Also, in subjects with schizophrenia, latent toxoplasmosis was associated with suicide attempts in younger but not older subjects (Okusaga et al. 2011). In contrast, two larger studies, but perhaps with less well defined diagnostic criteria, using data from the same dataset collected between 1988 and 1994, and limited to an age range between 20 and 39 years, did not find an association between depression and T. gondii (Pearce et al. 2012, Gale et al. 2014). Finally, a more recent study from New Zealand of 837 subjects found no association between seropositivity for toxoplasmosis and depression (Sugden et al. 2016).

Given the association between latent toxoplasmosis and behaviour including schizophrenia and the high prevalence of depression and latent toxoplasmosis, we sought to better clarify the association between latent toxoplasmosis and depression using a recently available large dataset representative of the U.S. population based on data collected between 2009 and 2012, consisting of subjects with a much wider age range than used by the previous three large studies. Finding results similar to prior studies but with utilisation of more recent data, with a wider age range and a different method of determining depression, would confirm prior results and add convergent validity.

\section{MATERIALS AND METHODS}

\section{Study sample}

We obtained cross-sectional data regarding depression, suicidal ideation, Toxoplasma gondii IgG seropositivity, sex, educational attainment, race-ethnicity, age, poverty-to-income ratio (PIR) and cigarette smoking in adults from the United States' Centers for Disease Control and Prevention's (CDC) 2009-2012 National Health and Nutrition Examination Survey (NHANES) datasets. The NHANES survey consists of various types of health-related and behavioural data (laboratory work, interview and physical examination) collected in the United States. Through weighted, multi-stage sampling, these data represent the U.S. population (Centers for Disease Control and Prevention 2012).

In the present study, data were available for 5487 adults between the ages of 20-80 years. We included only subjects who had laboratory work for anti-T. gondii IgG antibody titres and who also had completed the Patient Health Questionnaire 9 (PHQ-9), an assessment of depression (Kroenke et al. 2001, Kroenke and Spitzer 2002).

\section{Toxoplasma gondii IgG antibody}

NHANES laboratory staff assessed the presence of $T$. gondii IgG antibodies in blood serum by enzyme immunoassay. The process requires incubating samples to antigen-covered wells that immobilise any present antibodies. A conjugate and substrate are then applied and, if $T$. gondii antibodies are present, a colour change occurs, which can then be measured via spectrophotometer and compared to a standardised optical density curve to determine the antibody titre. We coded all IgG titre results at or above $33 \mathrm{IU} / \mathrm{ml}$ as positive for infection and all values below this point as negative for infection to create a binary $T$. gondii variable (Centers for Disease Control and Prevention 2016). We applied a natural $\log$ transformation to create a $T$. gondii IgG titre concentration variable to account for non-normality. Separate analyses were conducted for both $T$. gondii seroprevalence and $T$. gondii titre concentration.

\section{Depression}

Depression and its severity were assessed with the PHQ-9 (Kroenke et al. 2001, Kroenke and Spitzer 2002). The PHQ-9 is a nine-item checklist that assesses depression through questions such as, "Over the last 2 weeks, how often have you been bothered by the following problems: feeling down, depressed or hopeless?" The subject is then asked to choose from four possible response options: "Not at all," "Several days," "More than half the days," and "Nearly every day." The responses are coded from 0 to 3, respectively. A follow-up question on the PHQ-9 then asks respondents, "How difficult have these problems made it for you to do your work, take care of things at home, or get along with other people?" Response options for the last question are "Not difficult at all," "Somewhat difficult," "Very difficult" or "Extremely difficult." This last question is not scored but is used as a qualifier as it addresses whether symptoms impact social and/or occupational functioning, which is required for diagnosis. A tentative diagnosis of depression can be made by using the following criteria: if 1) a score of 2 or 3 on at least one of the following symptoms questions: feeling little interest or pleasure in doing things, feeling down, depressed or hopeless; 2) a score of 2 or 3 for five or more of the nine symptoms questions; and 3) subject report of 1 ("somewhat difficult") or higher on the follow-up question assessing how much the depression symptoms interrupted their social and/or occupational functioning. Thus, in our analysis, we only considered respondents that met this criterion to have probable depression and considered all other respondents to be non-depressed. This way, we were able to distinguish between those reporting symptoms of depressed mood versus those likely experiencing actual depression.

Question 9 of the PHQ-9 specifically assesses suicidal ideation through the question "In the last two weeks, how often have you thought that you would be better off dead or of hurting yourself in some way?" As with the other questions in the PHQ-9 assessment, subjects could choose from the options: "Not at all," "Several days," "More than half the days," and "Nearly every day." This question provided an opportunity to test the association between suicidal ideation and T. gondii seropositivity and anti-T. gondii IgG antibody titres. We included subjects with data for this question, regardless of overall PHQ-9 outcome, in all analyses that investigated associations with suicidal ideation.

\section{Control variables}

To control for potential confounding, we included a number of covariates in all analyses. Categorical variables included sex, education, race-ethnicity, and cigarette smoking. Continuous covariates included age and PIR. The education variable included categories for subjects who had achieved less than a high-school diploma, a high-school diploma or equivalent (e.g. through completion of the general education development exam) or greater than high-school education. Groups for the race-ethnicity variable 
included non-Hispanic white, non-Hispanic black, Hispanic and "other." PIR is defined as the ratio between household income and the federally established poverty threshold for household income, which varies by family size and geographic location, at the time of the NHANES survey. Thus, PIR is a continuous variable ranging from $0.00-5.00$ with values above 1.00 reflecting incomes increasingly higher than poverty while values below 1.00 reflect incomes increasingly lower than poverty. In the NHANES database, PIR values above 5.00 were coded as 5.00 for confidentiality reasons. Cigarette-smoking status was ascertained in the NHANES survey via two questions. The first question was whether or not the participants had smoked 100 or more cigarettes within their lifetime. Of those who answered "Yes", the follow-up question, "Do you now smoke?" was then asked. We considered all subjects who answered "Yes" to both questions as cigarette smokers and those who answered "No" to either or both questions as non-smokers.

\section{Statistical analyses}

We used Stata release 14.1 for all statistical analyses. Use of the built-in svy prefix enabled the inclusion of NHANES-generated cluster, strata, and weighting variables. The inclusion of these variables is recommended to account for the complex multi-stage sampling methods used in the NHANES.

We used logistic regression to examine the relationship between depression and T. gondii infection status and also to test for associations between depression and anti-T. gondii $\operatorname{IgG}$ antibody titre. We used natural-log transformed values of the anti-T. gondii IgG antibody titres to approximate a normal distribution. Because of the ordered, categorical nature of the suicidal ideation variable, we used ordered logistic regression to test the prediction of suicidal ideation from $T$. gondii infection status as well as T. gondii IgG titre.

We included sex, educational attainment, race-ethnicity, age, PIR and cigarette smoking status as control variables in each of the above models. We also tested for potential interactions between latent toxoplasmosis and age, PIR, gender, education level, race-ethnicity and cigarette smoking status in predicting depression. C-reactive protein (CRP) was only available for a subset of the sample included in the study (2009-2010 NHANES). We estimated some supplementary models on this reduced sample (n $=2933$ ) including CRP as an additional control and report the results below.

\section{RESULTS}

The overall number of subjects in the sample was 5487 . Table 1 includes weighted estimates from the NHANES survey describing the U.S. non-institutionalised population age 20 to 80 years for the years 2009-2012. Thirteen percent were seropositive for Toxoplasma gondii. Forty-five percent were male and the average age was 46.2 years $(\mathrm{SE}=0.57)$. The majority of U.S. adults was non-Hispanic white $(71 \%)$ and $62 \%$ had greater than a high-school education. Approximately eight percent of U.S. adults met criteria for probable clinical depression (Table 1), consistent with data on 12-month prevalence rates of about 7\% of Major Depressive Episode among U.S. adults in 2014 (U.S. Department of Health and Human Services 2014). Finally, an estimated five percent reported any amount of suicidal thoughts over the previous two weeks. Only $1 \%$
Table 1. Weighted summary statistics of U.S. adults 20-80 years old, National Health and Nutrition Examination Survey 2009-2012.

\begin{tabular}{lcc}
\hline & Percent (n) & SE \\
\hline Depression $^{\mathrm{b}}$ & & \\
PHQ-9 & $8 \%(439)$ & 0.48 \\
PHQ-9 and/or antidepressant use & $18 \%(1001)$ & 0.78 \\
Suicidal ideation & & \\
$\quad$ Not at all & $95 \%(5213)$ & 0.44 \\
Several days & $3 \%(164)$ & 0.35 \\
More than half the days & $1 \%(55)$ & 0.15 \\
$\quad$ Nearly every day & $1 \%(55)$ & 0.11 \\
Toxoplasma gondii seropositive & $13 \%(713)$ & 0.55 \\
Age (range 20-80) & $46.2^{\mathrm{a}}$ & 0.57 \\
Poverty to income ratio (range 0-5) & $2.84^{\mathrm{a}}$ & 0.06 \\
Male & $45 \%(2469)$ & 0.66 \\
Female & $55 \%(3018)$ & 0.66 \\
Race-ethnicity & & \\
$\quad$ Non-hispanic white & $71 \%(3896)$ & 2.57 \\
Non-hispanic black & $10 \%(549)$ & 1.19 \\
Hispanic & $13 \%(713)$ & 1.95 \\
Other & $6 \%(329)$ & 0.66 \\
Education & & \\
Less than high school & $16 \%(878)$ & 1.13 \\
High school or GED & $22 \%(1207)$ & 1.15 \\
$\quad$ More than high school & $62 \%(3402)$ & 1.78 \\
Cigarette smoking & $22 \%(1232)$ & 0.75 \\
\hline
\end{tabular}

${ }^{a}$ means calculated for continuous variables; ${ }^{b}$ two different methods were used to determine the presence of a depressive disorder: in the first operationalisation, a subject was determined to be depressed by the PHQ-9 diagnostic criteria alone, in the second operationalisation, the presence of depression was determined by the PHQ-9 diagnostic criteria or the by use of antidepressants; PHQ-9 - Patient Health Questionnaire 9; $\mathrm{SE}-$ Standard error; $\mathrm{N}=5487$.

of subjects indicated that they had suicidal thoughts nearly every day (Table 1).

We found no association between depression and latent toxoplasmosis regardless of whether we treated latent toxoplasmosis as a binary $(\mathrm{OR}=1.01,95 \% \mathrm{CI}=0.81-1.25$; $p=0.944)($ Table 2$)$ or continuous $(\mathrm{OR}=1.00,95 \%$ $\mathrm{CI}=0.96-1.06 ; p=0.868$ ) (Table 3 ) variable. In each of these models, both PIR and cigarette smoking independently predicted the presence of depression (Tables 2, 3). We further found that suicidal ideation was not significantly related to infection with $T$. gondii regardless of whether T. gondii was measured as binary (i.e. presence/absence) $(\mathrm{OR}=1.22,95 \% \mathrm{CI}=0.85-1.75 ; p=0.277)$ or continuous (i.e. titre levels) $(\mathrm{OR}=1.05,95 \% \mathrm{CI}=0.98-1.14$; $p=0.177$ ) (Tables 2, 3). If limited to depressed subjects only as determined by PHQ-9 criteria, there was still no significant association between $T$. gondii seropositivity and suicidal ideation.

Furthermore, there were no significant interactions between latent toxoplasmosis and any of the covariates (age, PIR, gender, education level, race-ethnicity, cigarette smoking status) in the analyses (Tables 2, 3). We also estimated reduced models where non-significant coefficients other than $T$. gondii were excluded; the results were substantively and statistically unchanged. Finally, in the NHANES 2009-2010 dataset, which included CRP but was a much smaller dataset, CRP was not a significant predictor of depression and there were no associations of 
Table 2. Logistic regression analysis of Toxoplasma gondii seropositivity predicting depression and suicidal ideation in U.S. adults National Health and Nutrition Examination Survey 2009-2012.

\begin{tabular}{|c|c|c|c|c|c|c|c|c|c|}
\hline & \multicolumn{3}{|c|}{ Depression: PHQ-9a } & \multicolumn{3}{|c|}{$\begin{array}{l}\text { Depression: PHQ-9 and/ } \\
\text { or antidepressant use }\end{array}$} & \multicolumn{3}{|c|}{ Suicidal ideation } \\
\hline & OR & $95 \% \mathrm{CI}$ & $p$ & OR & $95 \% \mathrm{CI}$ & $p$ & OR & $95 \% \mathrm{CI}$ & $p$ \\
\hline Toxoplasma gondii seropositive & 1.01 & {$[0.81,1.25]$} & 0.944 & 0.83 & {$[0.64,1.06]$} & 0.136 & 1.22 & {$[0.85,1.75]$} & 0.277 \\
\hline Male & 0.84 & {$[0.66,1.07]$} & 0.160 & 0.57 & {$[0.48,0.68]$} & 0.000 & 1.06 & {$[0.79,1.43]$} & 0.693 \\
\hline \multicolumn{10}{|l|}{ Education } \\
\hline Less than high school (ref) & - & - & - & - & - & - & - & - & - \\
\hline High school or GED & 0.75 & {$[0.53,1.06]$} & 0.102 & 0.93 & {$[0.72,1.20]$} & 0.579 & 0.80 & {$[0.47,1.36]$} & 0.400 \\
\hline More than high school & 0.80 & {$[0.58,1.13]$} & 0.196 & 0.96 & {$[0.70,1.31]$} & 0.778 & 0.67 & {$[0.45,1.00]$} & 0.052 \\
\hline \multicolumn{10}{|l|}{ Race-ethnicity } \\
\hline Non-hispanic white (ref) & - & - & - & - & - & - & - & - & - \\
\hline Non-hispanic black & 1.21 & {$[0.91,1.61]$} & 0.181 & 0.73 & {$[0.57,0.92]$} & .010 & 1.16 & {$[0.83,1.62]$} & 0.370 \\
\hline Hispanic & 0.90 & {$[0.66,1.24]$} & 0.511 & 0.62 & {$[0.47,0.80]$} & .001 & 1.54 & {$[1.00,2.37]$} & 0.050 \\
\hline Other & 1.07 & {$[0.63,1.83]$} & 0.797 & 0.62 & {$[0.43,0.90]$} & .012 & 1.03 & {$[0.52,2.02]$} & 0.939 \\
\hline Age & 1.00 & {$[1.00,1.01]$} & 0.334 & 1.01 & {$[1.01,1.02]$} & .000 & 1.00 & {$[0.99,1.01]$} & 0.481 \\
\hline Poverty to income ratio & 0.71 & {$[0.65,0.78]$} & 0.000 & 0.89 & {$[0.84,0.95]$} & .001 & 0.79 & {$[0.71,0.88]$} & 0.000 \\
\hline Cigarette smoking & 1.70 & {$[1.30,2.23]$} & 0.000 & 1.49 & {$[1.24,1.78]$} & .000 & 1.38 & {$[0.92,2.09]$} & 0.119 \\
\hline
\end{tabular}

$\mathrm{N}=5487$; ref - refers to the refernce group in the logistic regression; GED - General Education Development; OR - odds ratio, CI - confidence interval; PHQ-9 - Patient Health Questionnaire 9; a two different methods were used to determine the presence of a depressive disorder: in the first operationalisation, a subject was determined to be depressed by the PHQ-9 diagnostic criteria alone, in the second operationalisation, the presence of depression was determined by the PHQ-9 diagnostic criteria or the by use of antidepressants.

Table 3. Logistic regression analysis of Toxoplasma gondii $\operatorname{IgG}$ titre concentration predicting depression and suicidal ideation in U.S. adults National Health and Nutrition Examination Survey 2009-2012.

\begin{tabular}{|c|c|c|c|c|c|c|c|c|c|}
\hline & \multicolumn{3}{|c|}{ Depression: PHQ-9a } & \multicolumn{3}{|c|}{$\begin{array}{l}\text { Depression: PHQ-9 and/ } \\
\text { or antidepressant use }\end{array}$} & \multicolumn{3}{|c|}{ Suicidal ideation } \\
\hline & OR & $95 \% \mathrm{CI}$ & $p$ & OR & $95 \% \mathrm{CI}$ & $p$ & OR & $95 \% \mathrm{CI}$ & $p$ \\
\hline Toxoplasma gondii $\operatorname{IgG}(\mathrm{IU} / \mathrm{ml})$ & 1.00 & {$[0.96,1.06]$} & 0.868 & 0.94 & {$[0.89,1.00]$} & 0.036 & 1.05 & {$[0.98,1.14]$} & 0.177 \\
\hline Male & 0.84 & {$[0.66,1.07]$} & 0.160 & 0.57 & {$[0.48,0.68]$} & 0.000 & 1.06 & {$[0.79,1.43]$} & 0.696 \\
\hline \multicolumn{10}{|l|}{ Education } \\
\hline Less than high school (ref) & - & - & - & - & - & - & - & - & - \\
\hline High school or GED & 0.75 & {$[0.53,1.06]$} & 0.103 & 0.93 & {$[0.72,1.20]$} & 0.578 & 0.80 & {$[0.47,1.37]$} & 0.404 \\
\hline More than high school & 0.80 & {$[0.58,1.13]$} & 0.197 & 0.96 & {$[0.70,1.31]$} & 0.768 & 0.67 & {$[0.45,1.01]$} & 0.054 \\
\hline \multicolumn{10}{|l|}{ Race-ethnicity } \\
\hline Non-hispanic white (ref) & - & - & - & - & - & - & - & - & - \\
\hline Non-hispanic black & 1.21 & {$[0.91,1.61]$} & 0.181 & 0.73 & {$[0.57,0.92]$} & 0.011 & 1.16 & {$[0.83,1.62]$} & 0.376 \\
\hline Hispanic & 0.90 & {$[0.66,1.24]$} & 0.508 & 0.62 & {$[0.48,0.81]$} & 0.001 & 1.53 & {$[0.99,2.35]$} & 0.056 \\
\hline Other & 1.07 & {$[0.63,1.83]$} & 0.799 & 0.63 & {$[0.43,0.90]$} & 0.013 & 1.02 & {$[0.52,2.01]$} & 0.951 \\
\hline Age & 1.00 & {$[1.00,1.01]$} & 0.348 & 1.01 & {$[1.01,1.02]$} & 0.000 & 1.00 & {$[0.99,1.01]$} & 0.511 \\
\hline Poverty to income ratio & 0.71 & {$[0.65,0.78]$} & 0.000 & 0.89 & {$[0.84,0.95]$} & 0.001 & 0.79 & {$[0.71,0.88]$} & 0.000 \\
\hline Cigarette smoking & 1.70 & {$[1.30,2.23]$} & 0.000 & 1.49 & {$[1.24,1.79]$} & 0.000 & 1.38 & {$[0.92,2.09]$} & 0.120 \\
\hline
\end{tabular}

$\mathrm{N}=5487$; ref - refers to the refernce group in the logistic regression; GED - General Education Development; OR - odds ratio; $\mathrm{CI}$ - confidence interval; PHQ-9 - Patient Health Questionnaire 9; ${ }^{\text {a }}$ two different methods were used to determine the presence of a depressive disorder: in the first operationalisation, a subject was determined to be depressed by the PHQ-9 diagnostic criteria alone, in the second operationalisation, the presence of depression was determined by the PHQ-9 diagnostic criteria or the by use of antidepressants.

latent toxoplasmosis with depression and suicidal ideation after including CRP as a covariate.

Given the possibility that there could potentially be subjects who were currently being successfully treated for depression with medications who may have been included in our control group, we repeated each of the analyses with a broadened definition of depression that included subjects currently taking one or more of the antidepressants marketed in the United States regardless of whether they met criteria for depression based on the PHQ-9. This increased the percentage of subjects identified as depressed to $18.2 \%$. However, we again did not find a positive association between seropositivity for latent toxoplasmosis, measured as a binary variable, and depression $(\mathrm{OR}=0.83$,
$\mathrm{CI}=0.64-1.06 ; p=0.136$ ) (Table 2). However, defining depression by PHQ-9 criteria or current antidepressant medication use, we found an inverse association between anti- $T$. gondii $\mathrm{IgG}$ titre and depression $(\mathrm{OR}=0.94$, $\mathrm{CI}=0.89-1.00 ; p=0.036$ ) (Table 3 ). We found no interactions between $T$. gondii seropositivity and any of the covariates included in the present study with depression defined as meeting PHQ-9 criteria only or either current PHQ-9 criteria or current antidepressant medication use. Finally, we did not find any association between $T$. gondii seropositivity and suicidal ideation when limiting analysis to those subjects meeting criteria for depression through the PHQ-9 and who were also taking at least one antidepressant medication. 


\section{DISCUSSION}

In this follow-up study including more recent survey data, a wider age range and a different measure of depression compared to prior large studies (Pearce et al. 2012, Gale et al. 2014), we again failed to find a positive association between latent toxoplasmosis infection status or anti-T. gondii IgG antibody titre and depression or suicidal ideation. However, in the NAHANES survey, these data were specific to depression over the previous two weeks and thus may have excluded subjects who had had a previous depression or previous suicidal ideation in the recent or remote past. We attempted to partially address this problem by taking into account subjects who were currently taking antidepressant medication but still found no evidence of a positive association between latent toxoplasmosis and depression. In fact, in the analysis of anti- $T$. gondii IgG titres and subjects who met PHQ-9 or antidepressant-use criteria for depression, there was a negative association between latent toxoplasmosis and depression.

A recent meta-analysis found evidence for an association between $T$. gondii and schizophrenia, bipolar disorder, and addiction, but not major depression (Sutterland et al. 2015), consistent with our findings even though some of the depression studies included in the meta-analysis are difficult to compare to our findings because they included subjects with a restricted age range and depression with psychotic features. Sugden et al. (2016) studied a birth cohort of 837 participants at 38 years of age, when they determined $T$. gondii seropositivity and depression status. They found no association between major depression and latent toxoplasmosis $(\mathrm{OR}=1.01,95 \% \mathrm{CI}=0.66-1.54)$, although there was a trend for an association between latent toxoplasmosis and a history of suicide attempt that approached significance $(\mathrm{OR}=2.63,95 \% \mathrm{CI}=0.97-7.14$; $p=0.06)$.

Another recent study found an association between toxoplasmosis seropositivity and depression, but further analysis of the data demonstrated that having been scratched by a cat produced a much stronger association with depression than did seropositivity for latent toxoplasmosis (Flegr and Hodný 2016). The authors suggested that this stronger association with unipolar depression may be related to the presence of Bartonella henselae rather than T. gondii. Unfortunately, antibodies for $B$. henselae were not included in the NHANES data sets that we analysed.

In evaluating associations between latent toxoplasmosis and depression, it is important to not equate history of suicide attempts with depression. As noted, Arling et al. (2009) found that the difference in suicide attempts was not between those with and without depression but rather that among subjects with recurrent depression there were higher anti- $T$. gondii IgG antibody titres in those with a history of suicide attempt. In this regard, Yagmur et al. (2010) did not measure depression but rather assessed attempted suicide and compared controls with those that had attempted suicide. Similarly, Okusaga et al. (2011) found an association between latent toxoplasmosis and suicide attempts in subjects with schizophrenia. Although Sugden et al. (2016) did not find an association between latent toxoplasmosis and depression, the association between toxoplasmosis and suicide attempt was close to statistically significant $(p=0.06)$.

As a whole, the available findings suggest that any association between latent toxoplasmosis and mood disturbance could be less related to depression itself but perhaps more related to self-harm and/or impulsive behaviour (Ling et al. 2011). There is evidence for an association between latent toxoplasmosis self-harm including suicide attempts (Ling et al. 2011, Pedersen et al. 2012, Zhang et al. 2012). Finally, suicidal ideation and suicide attempts may well be two different phenomena, even though suicidal ideation is strongly associated with history of suicide attempts (Bebbington et al. 2010, Victor and Klonsky 2014). Unfortunately, the data set we used in the current study did not include information on prior suicide attempts, which is a limitation to this study.

Additional limitations of our study include its cross-sectional design. Further, although the PHQ-9 was used to assess depression status with the qualification that the depressive symptoms had to affect social/occupational function, these data were limited to symptoms over the past two weeks. Thus, the PHQ-9 identified only those subjects with current depression and as a result would have excluded subjects with a previous major-depressive episode and subjects who had recently been treated and reported normalised social/occupational function or significant reduction in symptoms. Thus, persons with longstanding history of depression but that were not currently depressed could have been in the control group.

We attempted to address this limitation by broadening our definition of depression to include subjects who were currently taking antidepressant medication but still found no positive association with latent toxoplasmosis. This broadened definition of depression has its own limitations in that antidepressants are often prescribed for conditions other than depression, such as anxiety, insomnia and chronic pain (Wong et al. 2016). Furthermore, these data were also quite limited in that we had no information on length of current depression, history of suicide attempts, history of self-injurious behaviours, or history of suicidal ideation.

Further, our inability to distinguish between depression subtypes in the NHANES datasets, such as bipolar depression or depression with psychosis, could have masked an association between latent toxoplasmosis and specific subtypes of depression. Despite these limitations, the current dataset has several strengths - large sample size, use of demographic covariates and investigation of potential interactions between latent toxoplasmosis and several covariates.

In conclusion, in the present study with an overall sample size of 5487, there was no evidence of an association between current depression (as assessed by the PHQ-9) and latent toxoplasmosis assessed by both seropositivity and anti- $T$. gondii IgG antibody titres concentration, nor was there any association between latent toxoplasmosis and current suicidal ideation. 


\section{REFERENCES}

Arling T.A., Yolken R.H., Lapidus M., Langenberg P., Dickerson F.B., Zimmerman S.A., Balis T., Cabassa J.A., Scrandis D.A., Tonelli L.H., Postolache T.T. 2009: Toxoplasma gondii antibody titers and history of suicide attempts in patients with recurrent mood disorders. J. Nerv. Ment. Dis. 197: 905-908.

Bebbington P.E., Minot S., Cooper C., Dennis M., Meltzer H., Jenkins R., Brugha T. 2010: Suicidal ideation, self-harm and attempted suicide: results from the british psychiatric morbidity survey 2000. Eur. Psychiatry 25: 427-431.

Berenreiterová M., Flegr J., Kuběna A.A., Neměc P. 2011: The distribution of Toxoplasma gondii cysts in the brain of a mouse with latent toxoplasmosis: implications for the behavioral manipulation hypothesis. PLoS ONE 6: e28925.

Carruthers V.B., Suzuki Y. 2007: Effects of Toxoplasma gondii infection on the brain. Schizophr. Bull. 33: 745-751.

Centers For Disease Control And Prevention 2012. National health and nutrition examination survey: survey methods and analytic guidelines. Services USDoHaH, Ed., Hyattsville, MD.

Centers For Disease Control And Prevention 2016. National health and nutrition examination survey: data documentation, codebook, and frequencies. Services USDoHaH, Ed., Hyattsville, MD.

Duffy A.R., Beckie T.M., Brenner L.A., Beckstead J.W., Seyfang A., Postolache T.T., Groer M.W. 2015: Relationship between Toxoplasma gondii and mood disturbance in women veterans. Mil. Med. 180: 621-625.

Fekadu A., Shibre T., Cleare A.J. 2010: Toxoplasmosis as a cause for behaviour disorders - overview of evidence and mechanisms. Folia Parasitol. 57: 105-113.

FLEGR J. 2015: Neurological and neuropsychiatric consequences of chronic Toxoplasma infection. Curr, Clin. Microbiol. Rep. 2: $163-172$.

Flegr J., Hodný Z. 2016: Cat scratches, not bites, are associated with unipolar depression - cross-sectional study. Parasit. Vectors 9: 8 .

Fuks J.M., Arrighi R.B., Weidner J.M., Kumar Mendu S., Jin Z., Wallin R.P., Rethi B., Birnir B., Barragan A. 2012: Gabaergic signaling is linked to a hypermigratory phenotype in dendritic cells infected by Toxoplasma gondii. PLoS Pathog. 8: e1003051.

Gale S.D., Brown B.L., Berrett A., Erickson L.D., Hedges D.W. 2014: Association between latent toxoplasmosis and major depression, generalised anxiety disorder and panic disorder in human adults. Folia Parasitol. 61: 285-292.

Hinze-Selch D., Daubener W., Eggert L., Erdag S., StoltenberG R., Wilms S. 2007: A controlled prospective study of Toxoplasma gondii infection in individuals with schizophrenia: beyond seroprevalence. Schizophr. Bull. 33: 782-788.

Kroenke K., Spitzer R.L. 2002: The phq-9: A new depression diagnostic and severity measure. Psych. Ann. 32: 1-7.

Kroenke K., Spitzer R.L., Williams J.B. 2001: The phq-9: Validity of a brief depression severity measure. J. Gen. Intern. Med. 16: $606-613$.
Ling V.J., Lester D., Mortensen P.B., Langenberg P.W., PosTOLACHE T.T. 2011: Toxoplasma gondii seropositivity and suicide rates in women. J. Nerv. Ment. Dis. 199: 440-444.

Okusaga O., Langenberg P., Sleemi A., Vaswani D., Giegling I., Hartmann A.M., Konte B., Friedl M., Groer M.W., Yolken R.H., Rujescu D., Postolache T.T. 2011: Toxoplasma gondii antibody titers and history of suicide attempts in patients with schizophrenia. Schizophr. Res. 133: 150-155.

Parlog A., Schluter D., Dunay I.R. 2015: Toxoplasma gondii-induced neuronal alterations. Parasite Immunol. 37: 159-170.

Pearce B.D., Kruszon-Moran D., Jones J.L. 2012: The relationship between Toxoplasma gondii infection and mood disorders in the third national health and nutrition survey. Biol. Psychiatry 72: 290-295.

Pedersen M.G., Mortensen P.B., Norgahd-Pedersen B., Postolache T.T. 2012: Toxoplasma gondii infection and self-directed violence in mothers. Arch. Gen. Psychiatry 69: $1123-1130$

Prandovszky E., Gaskell E., Martin H., Dubey J.P., WebSter J.P., MCCOnkey G.A. 2011: The neurotropic parasite Toxoplasma gondii increases dopamine metabolism. PLoS ONE 6: e23866.

Sugden K., Moffitt T.E., Pinto L., Poulton R., Williams B.S., CASPI A. 2016: Is Toxoplasma gondii infection related to brain and behavior impairments in humans? Evidence from a population-representative birth cohort. PLoS ONE 11: e0148435.

Sutterland A.L., Fond G., Kuin A., Koeter M.W., Lutter R., Van Gool T., Yolken R., Szoke A., Leboyer M., De HAAN L. 2015: Beyond the association. Toxoplasma gondii in schizophrenia, bipolar disorder, and addiction: systematic review and meta-analysis. Acta Psychiatr. Scand. 132: 161-179.

Torrey E.F., Bartko J.J., Lun Z.R., Yolken R.H. 2007: Antibodies to Toxoplasma gondii in patients with schizophrenia: A meta-analysis. Schizophr. Bull. 33: 729-736.

U.S. Department Of Health And Human Services 2014. Major depression among adults. N.I.O.H., National Institute Of Mental Health, http://www.nimh.nih.gov/health/statistics/prevalence/major-depression-among-adults.shtml, 03/2016.

Victor S.E., KlonsKy E.D. 2014: Correlates of suicide attempts among self-injurers: a meta-analysis. Clin. Psychol. Rev. 34 282-297.

Wong J., Motulsky A., Eguale T., Buckeridge D.L., Abrahamowicz M., TAmblyn R. 2016: Treatment indications for antidepressants prescribed in primary care in Quebec, Canada, 2006-2015. J. Am. Med. Assoc. 315: 2230-2232.

Yagmur F., Yazar S., Temel H.O., Cavusoglu M. 2010: May Toxoplasma gondii increase suicide attempt-preliminary results in Turkish subjects? Forensic Sci. Int. 199: 15-17.

Zhang Y., Traskman-Bendz L., Janelidze S., Langenberg P., Saleh A., Constantine N., Okusaga O., Bay-Richter C., Brundin L., Postolache T.T. 2012: Toxoplasma gondii immunoglobulin $\mathrm{g}$ antibodies and nonfatal suicidal self-directed violence. J. Clin. Psychiatry 73: 1069-1076. 